\section{Overdiagnosis during lung cancer screening: is it an overemphasised, underappreciated, or tangential issue?}

\author{
Frank C Detterbeck
}

The issue of overdiagnosis is frequently raised in the context of lung cancer screening. Two recent papers draw attention to this matter: one by Patz et al, ${ }^{1}$ which estimates the incidence of overdiagnosis in the experience of the National Lung Screening Trial, and the US Preventative Services Task Force report that recommends screening in an organised programme for individuals at an elevated risk of developing lung cancer. $^{2}$

Make everything as simple as possible, but not simpler (Albert Einstein)

Correspondence to Dr Frank C Detterbeck, Division of Thoracic Surgery, Department of Surgery, Yale University School of Medicine, PO Box 208062, New Haven, CT 06520-8062, USA;

frank.detterbeck@yale.edu
Overdiagnosis-what is it exactly? There are many definitions. In the Patz paper these are excess cancers identified in the screening arm of a randomised trial (either in general or among only screen-detected cancers). These excess cancers remain asymptomatic, by definition, since they would otherwise be detected in the non-screening arm during a follow-up phase ('catch-up period')—the duration of which is important. A more abstract definition is any excess cancer detected by screening that would not cause death if untreated, whether it becomes symptomatic or not. Others define it as cancer with a long volume doubling time (>400 days) during a period of observation, without addressing what might happen later. ${ }^{3}$ An existential view considers any cancer as overdiagnosed if the patient dies of other causes, regardless how aggressive the cancer was or how random the death might be (ie, being hit by a bus). ${ }^{45}$

The Patz study ${ }^{1}$ shows that, even with a single definition, the results vary dramatically depending on the perspective (figure 1). This underscores that overdiagnosis is not a simple concept.

A critical problem is that overdiagnosis can only be defined in retrospectlooking back in a population after a long period. Therefore the concept is abstract from a clinical point of view. The term 'overdiagnosis' is simply black and white -fine from a retrospective viewpoint. Yet the clinician has to make decisions prospectively about an individual patient, not a population. It is more useful clinically to frame the discussion in more nuanced terms such as indolent, less aggressive and more aggressive cancers. ${ }^{4}$ These terms leave some room for clinical judgment and for careful observation to see if a 'well behaved' lung cancer ever evolves to something that needs treatment.

\section{THE BABY AND THE BATH WATER}

Does the Patz paper diminish the value of lung cancer screening by showing that the 


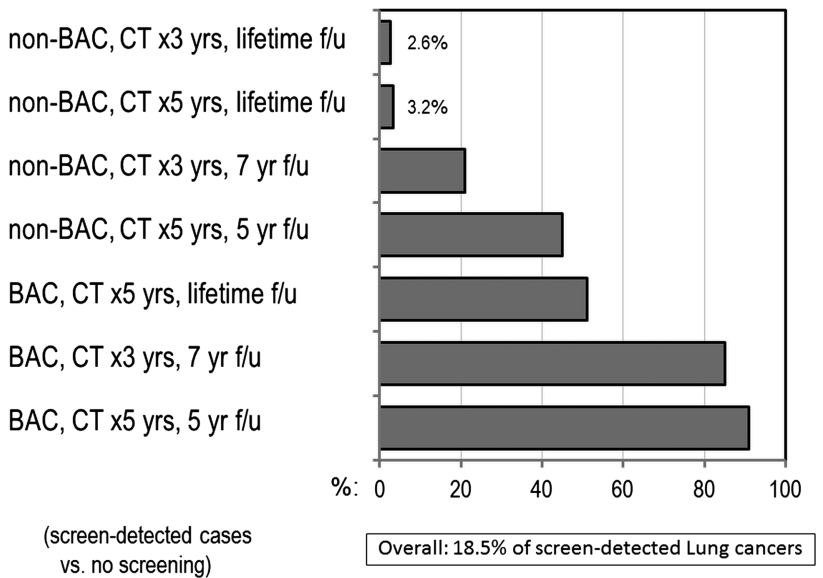

Figure 1 Proportion of overdiagnosed cases, according to histological type of cancer, screening and follow-up duration. Overdiagnosis is defined as the excess screen-detected cancers detected in a model of screening versus no screening. Taken from Patz et al. ${ }^{1}$ BAC, bronchioloalveolar carcinoma; f/u, follow-up; yr, year.

rate of overdiagnosis is substantial? It is true that detection of a cancer that is destined never to cause symptoms in that patient is of no benefit-to the patient or to the healthcare system. However, the reduction in mortality from lung cancer observed in the National Lung Screening Trial resulted from a reduction in the number of cases of lung cancer diagnosed in stage IV (33\% less in the low-dose computed tomography (LDCT) arm). This is true independent of whether there was a cohort of extra cancers which were not likely to progress to symptomatic tumours. Perhaps we should react more judiciously with some 'well behaved' cancers, but that does not impact the benefit of screening.

\section{TARGETS AND TANGENTS}

With respect to implementation of lung cancer screening, are we missing the point when we focus on potentially overdiagnosed cases? If reducing lung cancer mortality stems from reducing the number of cancers that are stage IV when diagnosed, shouldn't we focus on studying how we can impact the aggressive cancers the most? Are we too focused on the wrong end of the spectrum?

Are we missing the mark when we try prospectively to identify which cancers are 'overdiagnosed'? We know little about growth rates over long periods and can only extrapolate crudely from short periods of observation. Data from screening studies shows some variability and non-linearity of growth rates. ${ }^{6}$ Similarly, the models developed by Patz et al suggest that among the lung cancers with low aggressiveness (should we call them overdiagnosed?), in fact, some will become symptomatic-in the model $14 \%$ at 5 years and $27 \%$ at 10 years of bronchioloalveolar carcinomas (BACs) become symptomatic.

It may be more useful to accept that some screen-detected cancers will never become a threat and some will, and that we are not able to sort out which of the low grade tumours we can ignore forever. This allows us to focus instead on defining the right time to intervene. Are there signs that an initially 'well-behaved' lung cancer is gradually becoming more aggressive (eg, development of a solid component in previously pure ground glass opacity)? The screening experience has shown us that this transformation is a gradual process, so waiting until there is a reason to intervene is well justified.

\section{CONCLUSION}

The article by Patz et al is sophisticated, welldone and an important contribution. It shows how nuanced the concept of overdiagnosis is. Importantly, it presents the findings in a way that moves us towards clinical applicability. But there are leaps: (A) it is based on histology, which we can't fully define until after resection and (B) the correlation between radiographic appearance and histology is imperfect, especially with respect to the outdated term BAC. The study suggests we should probably be reacting even to some 'overdiagnosed' cases, given that $27 \%$ will become symptomatic within 10 years. This contradiction arises because overdiagnosis is a retrospective term.

Nevertheless, the Patz article is tangential to whether we should implement screening for lung cancer. It does not negate the benefits of screening; these accrue by decreasing stage IV lung cancer-a part of the spectrum not included in the analysis. To maximise benefits we need to understand the impact of details of screening on this end of the spectrum. However, the article does suggest how to minimise harms: intervening on non-BAC non-small cell lung cancer (NSCLC) is very rarely unnecessary (2-3\%), whereas for BAC we should be more circumspect, and wait for the right time to intervene. We should focus on clearly identifying when appropriate triggers for intervention have developed in these 'well behaved' lung cancers.

\section{Competing interests None.}

Provenance and peer review Not commissioned; internally peer reviewed.

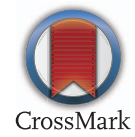

To cite Detterbeck FC. Thorax 2014;69:407-408.

Published Online First 19 March 2014

Thorax 2014;69:407-408.

doi:10.1136/thoraxjnl-2014-205140

\section{REFERENCES}

1 Patz EF Jr, Pinsky P, Gatsonis C, et al. Overdiagnosis in low-dose computed tomography screening for lung cancer. JAMA Intern Med 2014;174:269-74.

2 Moyer V. Screening for lung cancer: U.S. Preventive Services Task Force recommendation statement. Ann Intern Med 2014:160:330-38.

3 Henschke $\mathrm{Cl}$, Yankelevitz DF, Yip R, et al. Lung cancers diagnosed at annual CT screening: volume doubling times. Radiology 2012;263:578-83.

4 Detterbeck FC. Cancer, concepts, cohorts and complexity: avoiding oversimplification of overdiagnosis. Thorax 2012;67:842-5.

5 Reich JM. A critical appraisal of overdiagnosis: estimates of its magnitude and implications for lung cancer screening. Thorax 2008:63:377-83.

6 Detterbeck F, Gibson C. Turning gray: the natural history of lung cancer over time. J Thorac Oncol 2008;3:781-92.

7 Lindell RM, Hartman TE, Swensen SJ, et al. Five-year lung cancer screening experience: CT appearance, growth rate, location, and histologic features of 61 lung cancers. Radiology 2007;242:555-62. 